\title{
Pseudo Trust: Zero-Knowledge Authentication in Anonymous P2Ps
}

\author{
Li Lu, Member, IEEE, Jinsong Han, Member, IEEE, Yunhao Liu, Senior Member, IEEE, \\ Lei Hu, Jinpeng Huai, Member, IEEE, Lionel M. Ni, Fellow, IEEE, and Jian Ma
}

\begin{abstract}
Most trust models in Peer-to-Peer (P2P) systems are identity based, which means that in order for one peer to trust another, it needs to know the other peer's identity. Hence, there exists an inherent trade-off between trust and anonymity. To the best of our knowledge, there is currently no P2P protocol that provides complete mutual anonymity as well as authentication and trust management. We propose a zero-knowledge authentication scheme called Pseudo Trust (PT), where each peer, instead of using its real identity, generates an unforgeable and verifiable pseudonym using a one-way hash function. A novel authentication scheme based on Zero-Knowledge Proof is designed so that peers can be authenticated without leaking any sensitive information. With the help of PT, most existing identity-based trust management schemes become applicable in mutual anonymous P2P systems. We analyze the security and the anonymity in PT and evaluate its performance using trace-driven simulations and a prototype PT-enabled P2P network. The strengths of our design include the following: 1) no need for a centralized trusted party or Certificate Authority (CA); 2) high scalability and security; 3) low traffic and cryptography processing overheads; and 4) man-in-the-middle-attacks resistance.
\end{abstract}

Index Terms—Peer-to-Peer, authentication, mutual anonymity, trust, Zero-Knowledge Proof.

\section{INTRODUCTION}

A $\mathrm{S}$ an emerging model of communication and computation, Peer-to-Peer (P2P) networking has recently gained significant acceptance [1], [2], [3]. Most widely deployed P2P systems today, such as Gnutella, KaZaA, and BitTorrent, employ a routed-search-and-direct-download mechanism. Peers are linked in the overlay network, each maintaining several logical neighbors. Query flooding is the most popular search method used in such systems. If a peer receiving a query can provide the requested object, a response message is sent back to the requesting peer, and a direct download path is constructed between the downloader and the content provider.

One drawback of the above protocols is the fact that such P2P systems might compromise user privacy. The IP addresses of object requesters and providers can easily be discovered and translated into user names or postal addresses. Hence, many studies such as $\mathrm{P}^{5}$ [4] and APFS [5] focus on providing anonymous searching and downloading in P2P systems.

- L. Lu, J. Han, Y. Liu, and L.M. Ni are with the Department of Computer Science and Engineering, Hong Kong University of Science and Technology, Clear Water Bay, Kowloon, Hong Kong. L. Lu is also with the School of Computer Science and Engineering, University of Electronic Science and Technology of China, Chengdu 610054, P.R. China.

E-mail: \{luli, jasonhan, liu,ni\}@cse.ust.hk.

- L. Hu is with the State Key Laboratory of Information Security, Graduate University of the Chinese Academy of Sciences, Beijing 100049, P.R. China.E-mail: hu@is.ac.cn.

- J. Huai is with the State Key Lab of Software Developing Environment, Beihang University, Beijing 100083, P.R. China. E-mail: huaijp@buaa.edu.cn.

- J. Ma is with the Nokia Research Center, Beijing 100013, P.R. China. E-mail: jian.j.ma@nokia.com.

Manuscript received 31 May 2007; revised 28 Oct. 2007; accepted 28 Dec. 2007; published online 15 Jan. 2008.

Recommended for acceptance by G. Lee.

For information on obtaining reprints of this article, please send e-mail to: tpds@computer.org, and reference IEEECS Log Number TPDS-2007-05-0169. Digital Object Identifier no. 10.1109/TPDS.2008.15.
On the other hand, numerous concerns have been raised about the issue of providing authentic resources in P2P systems. To guarantee that real resources are received from authentic responders, some researchers have built trust models to help peers verify the validity of other entities [6], [7], [8]. Most trust models, however, are identity based, which means that for one peer to trust another, it needs to know the identity of the other peer. Thus, there exists an inherent trade-off between trust and anonymity in P2P systems. To the best of our knowledge, there is no existing $\mathrm{P} 2 \mathrm{P}$ protocol that provides mutual anonymity as well as trust management.

The purpose of designing an anonymous authentication protocol in $\mathrm{P} 2 \mathrm{P}$ systems is motivated by a specific problem: how to support authentication without exposing the real identities of peers. In this paper, we propose the design of the Pseudo Trust (PT) protocol, in which each peer generates an unforgeable and verifiable pseudonym using a one-way hash function. Such one-way mapping can effectively defend against impersonation and forgery, so that the pseudonyms can be used as the real IDs in P2P systems. This means that previous methods of identity-based trust management can be adopted. We also design a novel authentication scheme based on Zero-Knowledge Proof (ZKP) to help unfamiliar peers successfully complete authentication procedures during transactions. The salient features of PT include the following:

1. Achieving anonymity for authentication. PT enables pseudonym-based trust management so that the real identities of peers are protected during the authentication. PT can adopt current anonymous systems for anonymizing communications.

2. Realizing distributed trust. Previous trust management systems usually need a centralized trusted party, e.g., Certificate Authority (CA), to provide the trust for users. All users have to fully trust such an 
entity and share some secret with it. It risks leaking the user's private information and increases the system overhead. In contrast, PT allows users to generate their pseudo name individually and users do not depend on any third party to authenticate with each other.

3. Resisting man-in-the-middle-attacks (MIMAs). If we remove the centralized authority from the system, MIMA may severely threaten the authentication in distributed systems. A MIMA attacker can arbitrarily intercept, modify, or forge the message between two authentication parties so that the attacker can impersonate either of them to another party. PT, however, employs the one-way hash function to bind users' pseudonyms and the authentication paths together. Using the one-way hash function plus ZKP, users in PT not only authenticate the pseudo identities but also detect the authenticity of anonymous agents and check the integrity of messages. Our theoretical analysis shows the security of PT under MIMAs.

We discuss the implementation choices that were made for security and efficiency reasons and use the traces from DSS Clip2 to conduct trace-driven simulations to evaluate the parameter selections and the performance of our design. We also implement a PT prototype within a 50-machine overlay across the Internet in our laboratories in Beijing, Hong Kong, and other sites. Both our theoretical analyses and experimental results show that PT is effective, scalable, and completely decentralized with no need of a CA.

The rest of this paper is organized as follows: Section 2 introduces related works including trust management schemes, anonymous P2P protocols, and ZKP. Section 3 presents the PT design. Section 4 analyzes the security degree of PT. Section 5 presents our trace driven simulations and the performance evaluation. We introduce the PT prototype implementation in Section 6 and conclude this work in Section 7.

\section{Related Work}

In this section, we briefly describe related works in authentication, anonymity, and ZKP.

\subsection{Peer-to-Peer Trust and Authentication Schemes}

Abdul-Rahman and Halles propose a trust model and a recommendation protocol [9], focusing on decentralized systems. A prime and clear definition of trust is also provided. XREP [7] enables peers to evaluate and share other peer reputations by introducing a distributed polling algorithm. XREP also employs confirmation voting procedures among randomly chosen peers in order to prevent collusive cheating from cliques of malicious peers. The P-Grid focuses on an efficient data management technique to construct a scalable trust model for decentralized applications. EigenTrust [8] builds a virtual global matrix to represent individual reputations. NICE [10] provides a platform to implement distributed cooperative applications. Based on trust chains, NICE computes a user reputation in a PGP-like model. XenoTrust [11] provides a public infrastructure for a widearea trust computing environment. Generally, most P2P trust designs are identity based, where one peer does not trust another before knowing its identity.

\subsection{Anonymity}

Privacy has become an increasingly salient issue, and considerable progress has been made with anonymous communications [4], [5]. Several solutions achieve mutual anonymity for both initiators and responders in P2P systems, which generally aim at concealing the real identities of users during transactions. For example, in APFS [5], peers construct an anonymous path with tail nodes using an onion technique, providing complete and mutual anonymity for peers. Recent research has attempted to introduce reputation value into anonymous P2P systems or construct a trust management based on proxy techniques. However, failure to support authentication makes these approaches vulnerable to impersonation and MIMAs. Therefore, it is argued that providing privacy to peers increases the difficulties of authenticity and security. Obviously, there is a trade-off between authentication and anonymity.

\subsection{Zero-Knowledge Proof}

The purpose of ZKP protocols is to help a prover convince a verifier that she holds some knowledge (usually secret), without leaking any information about the knowledge during the verification process (zero-knowledge). The concept of ZKP was first introduced by Goldwasser et al. [12] and has since been employed in many authentication and identification protocols. Loosely speaking, a ZKP is an interactive proof system, which is comprised of a prover and a verifier. The principle rule is that the prover demonstrates knowledge of a secret to the verifier through several interactive rounds. During the process, the prover does not reveal any sensitive information to the verifier or any other parties. Each round involves a challenge (say, a question) from the verifier and a response (say, an answer) from the prover. If the secrets are related to user identities, ZKP can be used for identification and, in this case, is called Zero-Knowledge Proof of Identity (ZKPI). The security of ZKPI protocols is often based on the intractability of factoring large integers [12] or computing a discrete logarithm problem. Some have been improved to employ mutual authentication and key exchanges [13]. However, since almost all ZKP-based identification schemes are dependent on a trusted third party (such as a CA) as an authorized central server, they are not directly adopted by this design.

\section{Pseudo Trust}

The real and specific challenge that underlies the trade-off between trust and anonymity is that, on the one hand, all existing P2P trust systems attempt to link each peer ID with a trust value; on the other hand, anonymous designs hide the real IDs of communicating parties during transactions. This is where our proposed PT design enters the picture. Instead of using real IDs to deal with other peers in a P2P society, can peers use pseudonyms to interact with others and accumulate their reputations?

Clearly, if we would like to adopt such a mechanism, we need to guarantee that when a peer selects a pseudonym, it 


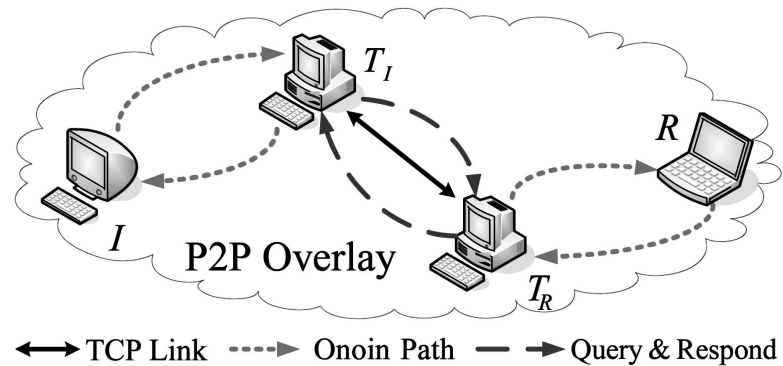

Fig. 1. PT query issuance and authentication.

is not likely to be a name already being used by another peer; and that pseudonym impersonations must be made impossible. That is, each peer is able to verify whether the other party it is communicating with is the real holder of the pseudonym it claims.

In this section, we first give an overview of the design of PT and then discuss its five key components, including Pseudo Identity Generation and Issuance, New Peer Initialization, Authentication and Session Key Exchange, File Delivery, and Trust and Reputation Management.

\subsection{Design Overview}

PT is applicable under most of today's widely deployed P2P protocols, such as Gnutella and KaZaA. For simplicity of discussion, we take a Gnutella-like P2P environment as a platform that PT runs on. In Gnutella, a requesting peer issues its queries in a flooding manner. A query is broadcast and rebroadcast until a certain criterion is satisfied. If the peer receiving the query can provide the requested object, a response message containing the IP address of the responder is sent back to the source peer along the reverse of the query path.

To protect real identities, in the PT design, each peer is required to generate a pseudo identity (PI) before joining the system. As illustrated in Fig. 1, peers construct anonymous onion paths and find tail nodes based on the APFS protocol [5]. Other selections of anonymous protocol designs are possible, but such changes are out of the scope of this discussion.

Before going into the details of PT, we list the notations we use in later discussions in Table 1.

\subsection{Pseudo Identity Generation and Issuance}

In PT, each peer is required to generate two items before joining the system: a pseudo identity (PI) and a pseudo identity certificate (PIC).

A $P I$ is used to identify and replace the real identity of a peer in a P2P system. This way, a peer does not have to expose its real identity when communicating with others. Furthermore, a peer's reputation is also coupled with its $P I$ instead of its real ID.

However, a naive $P I$ generation can lead to malicious impersonation. Thus, a $P I C$ is generated to authenticate the $P I$ holder. Whenever a $P I$ is issued, a PIC follows. A peer with a $P I C$ is able to verify whether the other party is the one it claims to be. The $P I$ and $P I C$ generation mechanism is described as follows: Terms not defined here can be found in [14].
TABLE 1

Notations of Variables

\begin{tabular}{cl}
\hline Notation & \multicolumn{1}{c}{ Specification } \\
\hline$I$ & Initiator of a query \\
$T_{A}$ & Tail node of peer $A[5]$ \\
$R$ & Responder \\
$f$ & Index of the requested file \\
$M$ & Malicious peer \\
$P I_{A}$ & The pseudo identity of peer $A$. \\
$P I C_{A}$ & Pseudo identity certificate of peer $A$ \\
$K$ & Session Key \\
$h_{i}$ & Hash function \\
$e$ & Challenge message \\
$k$ & Bit number of $e$ \\
\hline
\end{tabular}

Let $I D \in\{0,1\}^{*}$ denote the real identity of a peer $A$ $\left(\{0,1\}^{*}\right.$ denotes a set of binary strings). $Z_{n}^{*}$ is a multiplicative group of integers of modulo $n$.

PI and PIC Generation: Peer $A$ randomly chooses two large primes $p_{1}$ and $p_{2}$ and calculates the integer $n=p_{1} \times p_{2}$. PT then adopts a hash function, such as SHA-1. We slightly modify the prototype SHA- 1 and name the revisions $h_{i}$ to fit the different inputs and outputs. Here, PT first uses $h_{1}:\{0,1\}^{*} \times Z_{n}^{*} \times Z_{n}^{*} \rightarrow\{0,1\}^{m}$ to generate a Seed, where $m$ is the length of Seed, and $\{0,1\}^{*} \times Z_{n}^{*} \times Z_{n}^{*}$ is a Cartesian product. Peer $A$ then computes its Seed $_{A}$ by $\operatorname{Seed}_{A}=h_{1}\left(I D, p_{1}, p_{2}\right) \in\{0,1\}^{m}$.

Having $\operatorname{Seed}_{A}$, peer $A$ computes its $P I_{A}$ and $P I C_{A}$ as follows:

1. Choose $k$ distinct integers, $j_{1} \ldots j_{k}$. Compute $v_{j}=$ $h_{2}\left(\operatorname{Seed}_{A}, j_{i}, n\right)(\bmod n) \in Z_{n}^{*}$ for each small integer $j_{i}, i=1 \ldots k$, such that $v_{j}$ is a quadratic residue $(\bmod n)$, where $h_{2}$ is the hash function such that $h_{2}:\{0,1\}^{m} \times N \times N \rightarrow Z_{n}^{*}$ to generate a PIC, and $N$ is the set of positive integers.

2. Compute the smallest square root $s_{j}$ of $v_{j_{i}}(\bmod n)$. Here, we use $J=\left\{j_{i}\right\},\left\{s_{j_{i}}\right\}^{k},\left\{v_{j_{i}}\right\}^{k}$ to denote the sets of $j_{i}, s_{j_{i}}$, and $v_{j_{i}}$, respectively, where $i=1 \ldots k$. According to the Number Theory, it is computationally infeasible to compute square roots of modulo $n$ without knowing the factoring of $n$. The details can be found in [15, Section 6.6].

3. Compute $P I_{A}=h_{3}\left(\operatorname{Seed}_{A}, n\right) \in\{0,1\}^{m}$, where $h_{3}$ : $\{0,1\}^{m} \times N \rightarrow\{0,1\}^{m}$.

After the above operation, peer $A$ generates $P I C_{A}=$ $\left\{P I_{A}, n, J\right.$, Seed $\left._{A}\right\}$ and publishes its $P I C_{A}$ on public sites. Other peers can obtain the valid $P I C_{A}$ of peer $A$ from wellknown sites for later verification. To protect the authenticity of $n$, we combine each $P I$ with $n$ through hash functions. Therefore, whether or not the public sites are secured, PT becomes a "counterfeit-sensitive" protocol. For a detailed analysis on this part, see Section 4.7.

\subsection{New Peer Initialization}

After joining the P2P system, peer $A$ constructs anonymous sessions with existing peers using the APFS protocol. In this design, anonymous sessions are onion routes (ORs) comprised of chosen peers. A tail node $T_{A}$ acts as an agent relaying a message for peer $A . T_{A}$ and other peers in this path do not know $A$ is at the end point position. In APFS, 
peers use a multicast technique to send the query via a tail node to some servers anonymously, which is similar to the flooding procedure used by PT. To allow $T_{A}$ to send messages back to $A, T_{A}$ constructs the following $O R$ :

$$
O R_{A}=\left\{T_{A},\left\{\ldots A,\{m i x\} K_{A}, \ldots\right\} K_{T_{A}}\right\} .
$$

Meanwhile, peer $A$ builds another onion path $O R_{T_{A}}$ for sending message to $T_{A}$ anonymously:

$$
O R_{T_{A}}=\left\{X,\left\{\ldots T_{A},\{m i x\} K_{T_{A}}, \ldots\right\} K_{X}\right\} .
$$

After the anonymous session construction (note that each node selects its tail node and builds two onion paths), each peer can anonymously issue queries for the desired files. We use $I$ to denote an initiator. $I$ forwards a query $q$ for a certain requested file $f$, through $O R_{T_{I}}$ to its tail node $T_{I} . T_{I}$ then starts a flooding search in the P2P system.

When a peer receives the query and holds the requested file, it gets $I^{\prime}$ 's credit based on the trust management mechanism to help it decide whether to act as a responder $R$ and provide the file. If it decides to provide the file, it replies to this query through its tail node with a response including its tail node and the reputation record:

$$
I \stackrel{O R_{T_{I}}}{\longrightarrow} T_{I} \stackrel{\text { flooding }}{\longrightarrow} R: P I C_{I}, T_{I}, f \text {, request. }
$$

\subsection{Authentication and Session Key Exchange}

PT employs a modified ZKPI scheme. To adopt it into decentralized P2P networks, we remove the central authority servers in [16]. This scheme is based on the assumption that factoring a large integer is computationally infeasible.

When the query initiator, peer $I$ (note that $I$ is the pseudo identity of the initiator), receives multiple responses, it selects one or more peers with high reputations as potential downloader or responder. Without loss of generality, suppose $R$ is selected as one of the responders. Peer $I$ can initiate the authentication procedure to verify that the peer claiming to be the holder of $R$ is not lying. Peer $I$ sends an authentication request to $R$ through the anonymous path, $I \rightarrow T_{I} \rightarrow T_{R} \rightarrow R$ (note that $I \rightarrow T_{I}$ and $T_{R} \rightarrow R$ are onion paths $O R_{T_{I}}$ and $O R_{R}$, and $T_{I} \rightarrow T_{R}$ is a TCP connection). If $R$ decides to prove that it is the holder of pseudo identity $R$, it sends the reply with its $P I C_{R}$ to its tail node $T_{R}$ :

$$
R \stackrel{O R_{T_{R}}}{\longrightarrow} T_{R}: P I C_{R}, T_{R}, f, \text { response. }
$$

Following the APFS protocol, $T_{R}$ delivers the messages to $T_{I}$ directly through the TCP connection, and $T_{I}$ delivers the response to peer $I$ through $O R_{I}$ :

$$
T_{I} \stackrel{O R_{I}}{\longrightarrow} I: P I C_{R}, T_{R}, f, \text { response. }
$$

Having the $P I C_{R}$ of $R$, peer $I$ initiates the authentication procedure, as illustrated in Fig. 2. The authentication procedure includes two main phases: 1) peer $I$ acts as a prover to prove its validation to $R$ and 2) peer $R$ proves its authenticity to peer $I$ accordingly. These two phases are symmetrical and opposite in the proving direction. However, the order has been carefully chosen to avoid potential

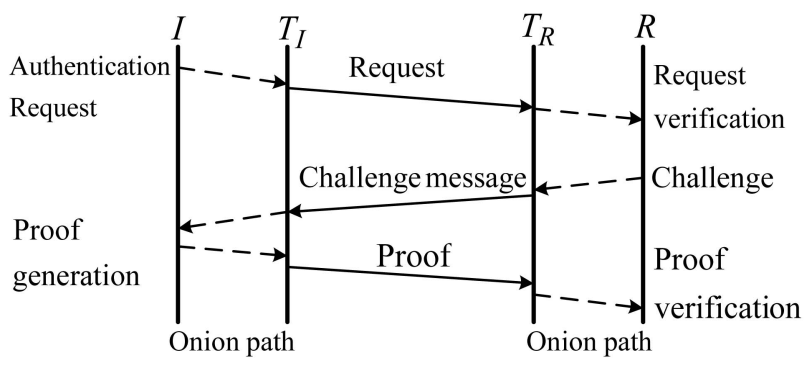

Fig. 2. Procedure of ZKP.

denial of service (DoS) attacks that may be launched onto $R$, which is further discussed in Section 4.

To provide confidentiality and integrity to data exchanges after authentication, we embed a Diffie-Hellman Key Exchange protocol into the authentication procedure to generate a session key held by $I$ and $R$ only. For efficiency purposes, we adopt a digital signature standard (DSS, see [17]) group to simplify the key exchange protocol. Three publicly known parameters $g, P$, and $Q$ are published by bootstrapping servers. $P$ (512 bits or longer) and $Q$ (160 bits) are prime numbers such that $Q$ divides $P-1$. $g$, satisfying $g^{Q}=$ $1 \bmod P$, is chosen from $(1, P-1)$ randomly. A detailed authentication procedure is described as follows:

1. Authentication Request: Before starting authentication, peer $I$ chooses two random numbers $x$ and $a$, where $x$ is a commitment for $R$ authenticating the $P I$ of $I$ in step 6 , and $a \in[1, Q)$ is used to generate the session key. $I$ chooses $c \in\left(0, n_{I}\right)$ randomly and computes $x=c^{2}\left(\bmod n_{I}\right), g^{a} \bmod P$ (for simplicity, we denote $g^{a} \bmod P$ as $\left.g^{a}\right)$, and $u=h^{4}\left(x, P I_{R}, T_{R}, g^{a}\right)$. I sends $\left\{x, u, g^{a}\right\}$ to peer $R$ and keeps $a$ as a secret. Here, $h_{4}$ is a hash function $h_{4}: Z_{n}^{*} \times\{0,1\}^{m} \times\{0,1\}^{*} \times Z_{p}^{*} \rightarrow$ $\{0,1\}^{k}$ to generate $u$, and $u$ is a $k$ bits long. We use $\left(u_{1} \ldots u_{i} \ldots u_{k}\right)$, where $u_{i} \in\{0,1\}$, to represent $u$.

2. Request Verification: Peer $R$ computes $u^{\prime}=h_{4}(x$, $\left.P I_{R}, T_{R}, g^{a}\right)$, then verifies whether $u=u^{\prime}$. If the verification holds, peer $R$ goes on to the next authentication step, otherwise it rejects this authentication request.

3. Peer $R$ checks whether $P I_{I}=h_{3}\left(\right.$ Seed $\left._{I}, n_{I}\right)$ holds. If not, peer $R$ terminates authentication. Otherwise, peer $R$ computes $\left\{v_{j_{i}}\right\}^{k}$ of peer $I$, $v_{j_{i}}=h_{2}\left(\operatorname{Seed}_{I}, j_{i}, n_{I}\right), j \in J_{I}, i=1 \ldots k$.

4. Challenge: Peer $R$ sends a random binary vector $e_{j}=\left(e_{j_{1}}, \ldots, e_{j_{k}}\right) \in\{0,1\}^{k}$ to peer $I$.

5. Proof generation: Peer $I$ sends the following to peer $R$ :

$$
y=c\left(\prod_{i=1}^{k} s_{j_{i}}^{e_{j_{i}}+u_{i}}\left(\bmod n_{I}\right)\right), s_{j_{i}} \in\left\{s_{j_{i}}\right\}_{I}^{k}, i=1 \ldots k .
$$

6. Verification: Peer $R$ checks (note that, here, $R$ uses its own $P I_{R}, T_{R}$ to compute the following result):

$$
y^{2}=x\left(\prod_{i=1}^{k} v_{j_{i}}^{e_{j_{i}}+u_{i}}\left(\bmod n_{I}\right)\right), v_{j_{i}} \in\left\{v_{j_{i}}\right\}_{I}^{k}, i=1 \ldots k .
$$


Peer $R$ accepts peer $I^{\prime}$ s proof if the equality holds, otherwise it rejects the proof. After peer $R$ verifies peer $I$, peer $I$ verifies $R$. Note that the above interactive communication is anonymous and the messages are relayed through onion paths and the TCP connection between the tail nodes $T_{I}$ and $T_{R}$.

The session key exchange scheme here deserves some discussion. When peer $R$ executes step 1 on its side, it picks a random number $b \in[1, Q)$ simultaneously and keeps $b$ as a secret. When the authentication is successfully completed, peer $I$ computes $K=\left(g^{b}\right)^{a} \bmod P$, and peer $R$ computes $K^{\prime}=\left(g^{a}\right)^{b} \bmod P$. Clearly, we have $K=K^{\prime}=g^{a b} \bmod P$, and therefore, peers $I$ and $R$ use $K$ as their session key for the subsequent file transmissions.

Here, 1) the length of $P I, 2)$ the length of $m, 3$ ) the large integer $n$, and 4) the number of quadratic residue $k$ are four key parameters. The selections of $m, n$, and $k$ are essential to the security degree of PT, on which we have more discussions in Section 4. Based on our analyses and experimental results, we choose $m \geq 64, n$ as a number being 1,024 bits long, and $k=80$.

\subsection{File Delivery}

After completing the authentication procedure, the two parties obtain a shared session key $K=g^{a b} \bmod P$. For confidentiality, PT employs a symmetric cipher algorithm such as AES to encrypt the content of a desired file using the key $K$. The integrity mechanisms are used to prevent the data from being forged, replaced, or modified during transmission without being detected, while the authenticity indicates that the receiver can be assured that the sender really did send that message (nonrepudiation). In this design, peers use a SHA- 1 hash function to generate a Message Authentication Code (MAC) as a warrant to convince the opposing party that the file is valid and guarantee the integrity of the data. For example, $R$ computes a MAC, $h(F \| K)$, using hash function $h(\cdot)$, then encrypts the file $F$ attached with the MAC into ciphertext $C$, and delivers $C$ to $I$, where $\|$ denotes the concatenation:

$$
R \stackrel{C=\text { Encrypt }_{K}(F \| h(F \| K))}{\longrightarrow} T_{R} \longrightarrow T_{I} \longrightarrow I .
$$

When peer $I$ receives $C$, it decrypts $F^{\prime}$ from this cipher and checks whether $h\left(F^{\prime} \| K\right)=h(F \| K)$. If they are equal, peer $I$ can be sure that $F$ is indeed the file sent from $R$ and $F$ has not been modified during transmission. If the file does not need to be encrypted, $R$ can simply compute $h(F \| K)$ and attach it with the file. A peer can make a flexible choice depending on its security requirements.

\subsection{Trust and Reputation Management}

After completing the authentication procedure, peer $I$ can download files from peer $R$. Similar to the authentication message exchange, the file can be delivered through anonymous sessions. A peer can either use the session key to encrypt the file or only encrypt the MAC of the file according to the users' security requirements. Since only peer $I$ and peer $R$ know their session keys, other peers, even the tail nodes, cannot forge the file to deceive the initiator during the data transmissions.
After downloading a file, peer $I$ can evaluate the file and provide comments to peers who provide resources such that most existing trust management mechanisms, such as EigenTrust [8] and XenoTrust [11], become applicable. The only difference after employing PT in these systems is that the reputation of peers will be connected with peer pseudo identities (PIs) instead of their real IDs or IP addresses.

\subsection{Pseudo Trust in Structured Peer-to-Peer System}

PT can also be adopted in structured P2P systems. In structured P2Ps, such as CAN and Chord, each peer's IP address and resource can be mapped to a point in the ID space using distributed hashing tables (DHTs). Each peer stores a fraction of the entire ID space, for example a zone in CAN. Lookup processes are handled as follows: On the one hand, the resource index is mapped to a point in the ID space via DHT by the resource holder, for example the Insert(key, value) process in CAN. The index, with the IP address of the resource holder, will be maintained by the peer whose zone covers the point. On the other hand, a requester computes the key of desired resource and issues it to DHT, for example the Lookup(key) process in Chord, to obtain the index of desired resource from the peer holding this information. Then, the requester will directly contact the provider to retrieve the resource. In this procedure, there is no anonymity protection for peers.

PT can be applied in structured P2P systems. First, the core technique of structured P2P systems is the consistent hashing function, which has features including the uniform distribution of outputs and resilience to collisions. These features are also essential properties of cryptographic hash functions ${ }^{1}$ [14], which have been employed in the PT for PI generation. The typical consistent hash function used in structured P2P systems is MD-5 or SHA-1. In PT, the PIs of users are also generated by using SHA-1 or MD-5, which enables PIs of PT to be used in structured P2P systems with slight modification. In addition, adopting PT in structured P2P system can enhance the anonymity of peers. Structured P2Ps such as CAN or Chord generate the peer's identity through DHTs but are "limited" to achieve anonymity. In most structured P2Ps, a peer's ID is derived by hashing the peer's IP address. Peers do not input any unique secret into the hash function to generate the IDs. Therefore, any peer who knows a given IP address can easily generate the corresponding ID. This process makes peers in CAN or Chord suffer from impersonation. It thereby degrades the anonymity and security and cannot guarantee the validation of authentication. In PT, peers use their unique secrets as an input of ID generation. It makes the pseudo ID verifiable and invulnerable to impersonation. By using PT authentication mechanism, the validation of a pseudo ID is guaranteed. Thus, replacing the original ID with a $P I$ via PT protocol will enhance the anonymity and the security in structured P2P systems. Second, instead of embedding an IP address in the resource index, the resource provider can preconstruct an onion path, which points to itself, and attach it to the resource index. In this

1. Loosely speaking, the cryptographic hash function is defined as the hash function with three properties: one-wayness, uniform and pseudorandom outputs, and collision resistance. 
case, any requester can utilize the onion path to contact the resource provider, while knowing nothing about the provider's identity. Of course, the initiator also employs an onion path and an anonymous agent for issuing the query to DHT, communicating with the peer holding the resource index, and retrieving the resource from the provider. In this way, PT's authentication mechanism can be adopted in structured P2Ps.

Some previous works have been done to anonymize structured P2P systems. Similar to unstructured P2Ps, anonymous approaches in structured P2Ps achieve anonymity via secured paths. The main principle of those schemes is to construct an anonymous path, predetermined by the initiator [18] or randomly probed during the message delivery [19]. For example, Salsa [20] presents an anonymous communication system based on DHT. To enhance the anonymity, each user constructs a circuit using Tor, which is in fact an anonymous path, to contact a proxy node. The proxy node performs lookup for the initiator, and hence, this proxy node is equivalent to a tail node, which is an anonymous agent, as we mentioned in Section 3.4. In other anonymous structured systems, most of them have such kind of anonymous paths, such that the nodes at the end of those paths can serve as the anonymous agents. Once assigning the anonymous agents, PT can be easily conducted over those systems. Therefore, structured P2P systems can seamlessly adopt PT.

\section{Security AnALysis}

We first analyze the anonymity degree of PT and, then, discuss the attack resilience of PT, such as the impersonation and MIMA.

\subsection{Anonymity}

PT uses a cryptographic hash function such as SHA-1 to generate $P I$ s from real identities for two reasons. First, the hash function is efficient and easy to use. Second, the hash function is publicly available for all peers in all networks with no need to exchange confidential information shared among them. These two properties are extremely appropriate for open P2P environments. We show the computation cost of $P I$ generation in Section 6 .

The anonymity of a peer's identity comes directly from the one-way property of cryptographic hash functions. Let $h(\cdot)$ be a hash function with $m$-bit-long hash values and assume it is well designed and has no structural drawback for cryptanalysis. In cryptograph terminology, $h(\cdot)$ takes advantage of a pre-image-resistance property, i.e., for any given hash value $y$, it is computationally infeasible to find an $x$ such that $h(x)=y$. Here, "infeasible" means we should do at least $2^{m-1}$ calculations of hash evaluation in general to find such an $x$ (see [21, Section 18.1]).

A malicious peer may launch advanced attacks, such as finding two different but real identities so that the two identities have the same $P I$. It might then use one of the two identities to impersonate the peer with the other identity. However, this kind of attack is withstood by the collision-resistance of hash functions: it is computationally infeasible to find a pair $(x, y)$ such that $h(x)=h(y)$. For a hash function with $m$ bit hash values, $2^{m / 2}$ calculations are required to find a collision with a probability of $1 / 2$, which is infeasible for $m \geq 128$ (see [21, Section 18.1]).

For $m=64$, finding the pre-image of this hash value needs 600,000 mips-year, while finding the collision of this hash value only needs $2^{32}$ calculations, which can be executed in 1 mips-h (more details can be found in [21, Section 7.2]). Hence, the hash value length should be more than 64 bits. In the PT design, for properly executing the ZKP (see Lemma 2) and obtaining high overall security, we suggest $m \geq 80$. Proper hash functions for our design include SHA-1 $(m=160)$ and its offspring. If we choose SHA-1, a malicious peer must take $2^{159}$ calculations to work out the identity from the $P I$ and $2^{80}$ calculations to find a pair of inputs with the same output. These are obviously infeasible computations. Thus, PT achieves complete anonymity for peers in networks. Malicious peers cannot deduce a real identity from a $P I$. In other words, the PT scheme provides a secure conversion from real identities to anonymous $P I$ s.

Note that cryptanalysis is making surprisingly excellent progress in cracking widely used hash functions. However, the recent common view is that these functions are still cryptographically secure for applications. In PT, we employ the hash function SHA-1.

\subsection{Impersonation}

PT can effectively defend against impersonation. If PT is executed successfully between the initiator $I$ and responder $R, I$ (or $R$ ) does not leak any secret $\left\{s_{j}\right\}^{k}$ used in the authentication procedure to the opposite side and any adversary (i.e., PT is zero-knowledge). Without the secret, none can impersonate others, i.e., PT would not be subject to impersonation. We prove this feature below.

Theorem 1. Assuming factoring a composite number $n$ is intractable, if initiator $I$ and responder $R$ properly follow the PT protocol, then $R$ always accepts $P I$ of $I$ as valid, and vise versa. Meanwhile, $I$ or $R$ would not leak any knowledge of the secret $\left\{s_{j}\right\}^{k}$ to the opposite side and any malicious peer $M$. If a malicious node $M$ plays an impersonation, the successful probability is $\max \left(2^{-k}, 2^{-t}\right)$, where $k$ is the length of a challenge message e (in PT, $k=80$, on which we have more discussions later), and $t$ is an integer dependent on $n$ only. Especially, when $|n|=1,024$ bits, $t=87$.

Proof. First, we prove PT has the properties of completeness and soundness. In cryptology, loosely speaking, completeness is defined as the ability of the verifier to accept true statements by the prover, while soundness asserts that the verifier cannot be "tricked" into accepting an invalid statement from a false prover.

Lemma 1 (Completeness). If peers I and $R$ properly follow the authentication procedure, then peer $R$ always accepts the $P I$ of peer $I$ as valid.

Proof. According to the authentication procedure steps in Section 3.4, if peer $I$ owns the correct secrets $\left\{s_{j}\right\}^{k}$, it calculates a valid answer using the data $c$ received from peer $R$ :

$$
y=c\left(\prod_{i=1}^{k} s_{j_{i}}^{e_{j_{i}}+u_{i}}\left(\bmod n_{I}\right)\right), i=1 \ldots k
$$


Peer $R$ verifies the answer $y$ as follows:

$y^{2}=\left(c \prod_{i=1}^{k} s_{j_{i}}^{e_{j_{i}}+u_{i}}\right)^{2}=c^{2} \prod_{i=1}^{k}\left(s_{j_{i}}^{2}\right)^{e_{j_{i}}+u_{i}}=x \prod_{i=1}^{k} v_{j_{i}}^{e_{j_{i}}+u_{i}}\left(\bmod n_{I}\right)$.

Thus, peer $R$ always accepts the $y$ generated by $I$ if peer $I$ has the proper secrets $\left\{s_{j_{i}}\right\}^{k}$.

Similarly, peer $I$ always accepts the $P I$ of peer $R$ as valid, if $I$ and $R$ properly follow the authentication procedure. The proof is the same as above.

Lemma 2 (Soundness). Assume it is computationally infeasible for factoring $n$ and a malicious peer $M$ does not have any partial knowledge of the initiator's secret $\left\{s_{j_{i}}\right\}, i=1 \ldots k$. Suppose $M$ interacts the PT protocol with responder $R$ to impersonate initiator $I$ and convince $R$ that it is $I$. Then, the probability that $M$ succeeds is $\max \left(2^{-k}, 2^{-t}\right)$.

Proof. The ZKP protocol is based on the intractability of finding the square roots of modulo $n$. According to the number theory, finding such roots is equivalent to factor $n$ (See [22, Fact 3.46]).

Meanwhile, according to recent progress on number theory, the complexity of the best algorithm for factoring a generic number $n$ is $O\left(2^{t}\right)$ [23], where $t=$ $a(\ln n)^{1 / 3}(\ln \ln n)^{2 / 3} / \ln 2$ and $a \approx 1.93$. When $n \approx 2^{1024}$, $t=87$.

On the other hand, $M$ can cheat $R$ by first guessing the binary vector $e_{j}$ and then sending $x=$ $c^{2} \prod_{i=1}^{k} v_{j_{i}}^{e_{j_{i}}+u_{i}}\left(\bmod n_{I}\right)$ as well as $y=c\left(\bmod n_{I}\right)$ to $R$ to impersonate $I$. The success probability of guessing $e_{j}$ is $2^{-k}$ since the vector is $k$-dimensional. $M$ may try to increase this probability as follows: $M$ chooses an $x$ such that for a nonnegligible probability it can compute the square roots $y^{\prime} / y^{\prime \prime}$ of $x /\left(\prod_{i=1}^{k} v_{j_{i}}^{e_{j_{i}}+u_{i}}\right)$ $\left(\bmod n_{I}\right)$ for two vectors $e^{\prime}$ and $e^{\prime \prime}$. Then, the value $y^{\prime} / y^{\prime \prime}$ is calculated as follows:

$$
\begin{aligned}
y^{\prime} / y^{\prime \prime} & \left.=\left(\frac{x /\left(\prod_{i=1}^{k} v_{j_{i}}^{e_{j_{i}}^{\prime}+u_{i}}\right)}{x /\left(\prod_{i=1}^{k} v_{j_{i}}^{e_{j_{i}}^{\prime \prime}}+u_{i}\right.}\right)\right)^{\frac{1}{2}}\left(\bmod n_{I}\right) \\
& =\prod_{i=1}^{k} s_{j_{i}}^{e^{\prime \prime}+e_{j_{i}}^{\prime}}\left(\bmod n_{I}\right) .
\end{aligned}
$$

$y^{\prime} / y^{\prime \prime}\left(\bmod n_{I}\right)$ is the square root of $\prod_{i=1}^{k} v_{j_{i}}^{e_{j_{i}}^{\prime \prime}+e_{j_{i}}^{\prime}}\left(\bmod n_{I}\right)$. Thus, $M$ can compute the square roots in $Z_{n_{I}}^{*}$ within a polynomial time. As mentioned before, finding square roots in $Z_{n_{I}}^{*}$ is exactly equivalent to factor $n_{I}$, product of two primes. Therefore, we now have a method to solve the NP problem of factoring a composite number, which is obviously intractable in a polynomial time.

In summary, the probability that $M$ successfully convinces peer $R$ that it is peer $I$ is $\max \left(2^{-k}, 2^{-t}\right)$.

Similarly, if $M$ interacts the PT protocol with initiator $I$ to impersonate responder $R$ and convince $I$ that $M$ is $R$, then the probability that $M$ succeeds is $\max \left(2^{-k}, 2^{-t}\right)$. The proof is the same as above.

Now, we prove that PT would not leak any knowledge of the initiator and responder in the authentication procedure. In other words, PT is a ZKP protocol.

Lemma 3 (Zero-knowledge). PT is a ZKP protocol.

Proof (Sketch). Due to the page limitation, we just give a nonrigorous proof sketch. In PT, no information is revealed whatsoever $I^{\prime}$ s secret $\left\{s_{j}\right\}$ is. This is because the random number $x$ consists of random squares, and $I$ 's proof $y$ contains an independent random variable, which masks the values of $\left\{s_{j}\right\}$. Hence, all the messages sent from $I$ to $R$ are in the pattern of random numbers indistinguishable with the uniform distributions. That is, $R$ cannot get any information about $I$ 's secret $s_{j}$, which means PT is a ZKP protocol. For formal and detailed proof, we refer the readers to [16].

In summary, PT successfully performs authentication between honest initiator $I$ and responder $R$ and effectively defends against the impersonation attack. Therefore, we have proved Theorem 1, which claims that PT is provably secure under the impersonation attack. $\square$

\subsection{Replay Attack}

For a replay attack, malicious peers collect some previous proofs of an initiator and resend these proofs to the responder. To convince the responder, malicious peers must guess the challenge message $e$ (see Section 3.4) generated by the responder completely and correctly. According to the proof of Lemma 2, the probability of a malicious peer's guessing correctly a challenge message $e$ chosen by the responder is $2^{-k}$. Thus, the success probability of a replay attack is $2^{-k}$.

\subsection{Man-in-the-Middle-Attack}

A MIMA is an attack in which an intruder $M$ is able to arbitrarily access and modify messages between two parties without either party knowing that the link between them has been compromised. As a result, $M$ can successfully impersonate the initiator to the responder, or vice versa. To PT users, intruders can modify and relay the forged authentication messages to participants and try to convince peer $I$ or peer $R$ that $M$ is the opposing party. We define a MIMA to be successful if a malicious peer $M$ is able to convince peer $I$ or peer $R$ that $T_{M}$, which is indeed the tail node of $M$, is $T_{I}$ or $T_{R}$, a tail node of peer $I$ or peer $R$. We also assume $M$ is able to intercept, replace, and modify the messages arbitrarily.

As shown in Fig. 3, $M$ impersonates two victims simultaneously, which is challenging to defend and a key issue in our discussion. Such a MIMA is based on two possible instances. Instance 1: peer $R$ does not receive peer $I^{\prime}$ 's query $q$. Instance $2: R$ receives $I^{\prime}$ s $q$.

For Instance 1, since $R$ does not receive $q, R$ does not respond. In this case, to cheat $R, M$ has to 1) forward peer $I^{\prime}$ 's query $q$ directly to $R$ or 2 ) forge a $q^{\prime}$ and send it to $R$.

For item 1, $M$ acts like other relaying nodes in the transmission. Since $M$ does not modify anything, $R$ 


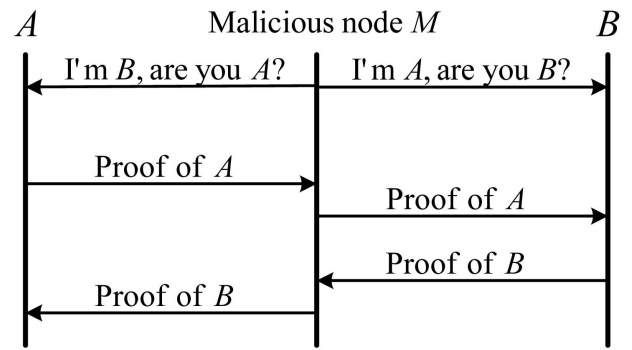

Fig. 3. MIMA attack. $M$ cheats $A$ or $B$ that $M$ is the real opposing party.

connects with $T_{I}$ through $T_{R}$ directly. Thus, $M$ cannot cheat anyone.

For item 2, the possible modification on $q$ by $M$ leads to two subcases: 1) $M$ modifies or just replaces the $P I C_{I}$ with its $P I C_{M}$ in $q$ such that $R$ considers $M$ as an initiator. This is useless for $M^{\prime}$ s attack because it would fail in the later verification without a valid $P I_{I}$. Otherwise, $M$ may hope to find a valid $P I C_{M}$ with the same hash value as $P I_{I}$, which is computationally infeasible as we discussed in Theorem 1. 2) $M$ modifies $q$ into $q^{\prime}=\left(P I C_{I}, T_{M}, f\right)$, as the point (1) shown in Fig. 4. The following discussion is based on this situation.

After receiving $q^{\prime}, R$ replies to $I$ with $\left(P I C_{R}, T_{R}, f\right)$. $M$ intercepts this reply, modifies this message to $\left(P I C_{R}, T_{M}, f\right)$, and delivers it to $I$. Note that $M$ has to modify $T_{R}$ to $T_{M}$, otherwise $I$ would ask $T_{I}$ to contact $T_{R}$. That is, $T_{M}$ is not involved in the authentication and the attack fails. See point (2) in Fig. 4.

Following step 1 in the authentication procedure, peer $I$ randomly chooses $c$ and $a$ and computes the commitment $x=c^{2} \bmod n_{I}, g^{a} \bmod P$, and $u=h_{4}\left(x, P I_{R}, T_{M}, g^{a}\right)$. Then, $I$ sends them back to $T_{M}$.

Upon intercepting this message, $M$ has only two choices of how to continue its intruding actions:

1. $M$ relays this message to $R$ without modification. Then, $R$ computes $u^{\prime}=h_{4}\left(x, P I_{R}, T_{R}, g^{a}\right)$ and checks it with the $u$ peer $R$ just received. According to the pseudorandom feature of the hash function, we have $u \neq u^{\prime} . R$ terminates the authentication procedure, and the attack fails. See point (3) in Fig. 4.

2. $M$ computes $u^{\prime \prime}=h_{4}\left(x, P I_{R}, T_{R}, g^{a}\right)$ and sends it to $R$. In such a case, $u^{\prime \prime}=u^{\prime}$. $R$ continues authentication. $R$ then sends a challenge $e$ to $T_{M}$. $M$ cannot know $e$ in advance and the best choice for $M$ is to deliver the challenge to $I$. Otherwise, $M$ can only guess $e$ with a probability of $2^{-k}$.

In choice 2, $M$ cannot modify $x$ and $g^{a}$ to make $u=u^{\prime}$. Due to the collision-resistance property of the welldesigned hash functions, finding such $x^{\prime}$ and $g^{a^{\prime}}$ that makes $h_{4}\left(x, P I_{R}, T_{M}, g^{a}\right)=h_{4}\left(x^{\prime}, P I_{R}, T_{R}, g^{a^{\prime}}\right)$ is computationally infeasible.

Peers $I$ and $R$ continue the authentication procedure following the PT protocol until step 5. At this point, peer $I$ generates a proof $y=c\left(\prod_{i=1}^{k} s_{j_{i}}^{e_{j_{i}}+u_{i}}\right)\left(\bmod n_{I}\right)$ and sends it back. Since the secret $\left\{s_{j_{i}}\right\}^{k}$ of $I$ is unknown to $M, M$ cannot forge a proof to pass $R^{\prime}$ s verification. If $M$ changes $e$ so as to pass the verification, it must guess the value of $e$ before $R$

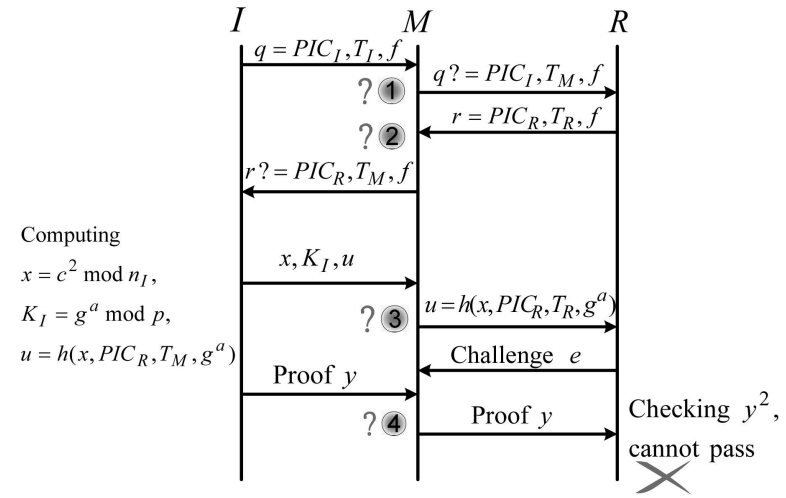

Fig. 4. Resistance from the MIMA.

generates $e$ and change the value of $y$ accordingly. Since the probability of such a successful guess is $2^{-k}$, it is infeasible. See point (4) in Fig. 4.

In step $6, R$ checks whether $y^{2}=x \prod_{i=1}^{k} v_{j_{i}}^{e_{i}}+u_{i}^{\prime}\left(\bmod n_{I}\right)$ holds. According to the previous discussion, $u=$ $h_{4}\left(x, P I_{R}, T_{M}, g^{a}\right)$, but $u^{\prime}=h_{4}\left(x, P I_{R}, T_{R}, g^{a}\right)$. It is clear that $u \neq u^{\prime}$. Thus, $R$ stops the authentication.

If $M$ attempts to continue cheating $I$ by impersonating $R$, since $M$ does not know the secret $\left\{s_{j_{i}}\right\}^{k}$ of $R$, it cannot pass the verification on $I^{\prime}$ s side. Thus, MIMA attempts made by intruder $M$ in instance 1 fail.

For Instance 2, $R$ receives $I^{\prime}$ s query $q$. In this case, $R$ has multiple queries containing an identical $P I$ with different tail nodes. Aware of being under attack, $R$ can simply discard the query or randomly select one of them to initiate the authentication procedure. The remaining analysis is similar to the case in Instance 1.

The key point of the authentication technique in PT is that we bind the proof, the tail node's information, and the key exchange data together with a peer's PI. With this design, any attempts to modify the identity messages cannot pass the verification of genuine protocol participants. Thus, MIMA fails to attack our proposed PT protocol.

\subsection{Collaborated Attack}

In practical P2P systems, subverted nodes may collaborate to enact identity fraud activities. Combined with the reputation auditing design, $\mathrm{PT}$ is also effective in defending against such collaborated attacks.

There are two extremely challenging collaborative attacks: 1) $I$ (or $R$ ) collaborates with intruder $M$ and 2) the tail node $T_{I}$ (or $T_{R}$ ) collaborates with $M$.

When $I$ (or $R$ ) is a co-conspirator that assists the malicious peer $M$, it provides its secret $\left\{s_{j_{i}}\right\}^{k}$ to $M$. $M$ can impersonate $I$ (or $R$ ). However, after authentication, $I$ (or $R$ ) must be responsible for its bad behavior. Therefore, collaboration with $M$ leads to $I$ (or $R$ ) losing reputation.

Another possible situation is when the tail node $T_{I}$ (or $T_{R}$ ) acts as a collaborator to help the intruder to provide a forged file. In this case, $T_{I}$ (or $T_{R}$ ) acts in a normal manner when $I$ and $R$ authenticate each other. This would relay all messages for $I$ and $R$ without modifying them until the authentication procedure completes. It would, however, replace the original file delivered from $R$ to $I$ with a forged one. Since PT combines a session key exchange 
procedure with the authentication phase, the confidentiality and integrity of the file can be protected by the session key $K=g^{a b} \bmod P$. Therefore, the tail nodes cannot substitute a forged file to cheat peer $I$.

\subsection{Pseudo Identity Security in Unsafe Public Sites}

As mentioned in this paper, the pseudo identity $P I$ and pseudo identity certificate $P I C$ of each peer are published on some well-known Websites. There exists a security problem, which may jeopardize the publisher. If a powerful adversary cracks a Website, which publishes the $P I$ s and $P I C$ s of some peers, the adversary would change some $P I^{\prime}$ s moduli $n$ to another one of which he holds the factors. Thus, the adversary can forge the values used in the authentication procedure to impersonate these cracked PIs. Fortunately, the technique used in the $P I$ generation can resist this attack. PT allows a pseudo identity $P I$ to be generated from the moduli $n$ and a seed through a hash function. Based upon the collision-resistance property of the hash function, $P I$ becomes the MAC of moduli $n$ and protects moduli $n$ from being forged.

When an adversary forges the moduli $n$ of a $P I$, he has two choices: 1) computing $P I^{\prime}=h_{3}\left(\right.$ Seed,$\left.n^{\prime}\right)$ and replacing $(P I, n)$ with $\left(P I^{\prime}, n^{\prime}\right)$ or 2$)$ just replacing $n$ with $n^{\prime}$.

Case 1: Since our scheme is pseudo identity based, this attack would not affect the owner of $P I$. In fact, this attack is equivalent to publishing a new pseudo identity of the adversary.

Case 2: According to the generation of $P I$, a pair $\left(n, n^{\prime}\right)$ satisfying $P I=h_{3}($ Seed,$n)=h_{3}\left(\right.$ Seed,$\left.n^{\prime}\right)$ is called a collision of $h_{3}$. Due to the collision-resistance property of the hash function (see Section 4.1), the probability of finding such $n^{\prime}$ is $2^{-m / 2}$ (where $m$ is the length of $P I$ ).

In summary, we manage to defend against the forging of moduli $n$ by binding $P I$ with $n$ through a hash function.

\subsection{Comparison to Public Key Infrastructure-Based Authentication Protocols}

In some applications, authentication systems are implemented based on an asymmetric cryptographic algorithm, such as RSA. There are two application models in the Public Key Infrastructure (PKI): CA-based and non-CA-based. The majority of PKI systems need centralized trust servers. In those applications, a commonly trusted third party, namely $\mathrm{CA}$, is required to ensure the validity of the binding of a user public key and her identity. By doing so, users usually provide signatures as well as their certificates to pass one another's verification. However, this CA-based model is not suitable for decentralized P2P environments.

To adopt PKI, anonymous systems remove CA. Users simply use their public keys as the pseudonyms. Further, self-signed certificate is used to authenticate the reality of a peer's pseudonym. This technique can provide anonymity for vender and vendee in a "face-to-face" scenario. In the complicate decentralized networks, however, this technique cannot resist the MIMA. For example, when Alice is going to download a file $f$, she first performs a search in the network. As we presented in Section 4.4, an attacker in the middle of communication between two transaction participants can impersonate them without being disclosed. Due to the lack of the knowledge of communication channels, current anonymous systems are subject to the MIMA attack. The main reason is that they do not bind the knowledge of communication channels with the authentication messages. Therefore, a MIMA attacker can arbitrarily relay the messages and impersonate the transaction participants.

Compared to them, PT handles the MIMA attack better, as we discussed in Section 4.4. Both the initiator and responder embed the knowledge of the path in the exchanged messages. After verifying the combined message's validation, transaction participants can detect the impersonation if it happens.

\subsection{Denial of Service Attack}

DoS attack has gained increasing concern in distributed networks. Typically, this attack renders networks, hosts, and other victim systems unusable by consuming the bandwidth of victim networks or deluging them with a huge number of requests to overload their systems. In the design of PT, the system may suffer from DoS attacks during authentication interactions. Since $I$ chooses a desired responder from the returned responses to start an authentication procedure, $I$ does not suffer from DoS attacks. Thus, we simply focus on the possibility that responders are under DoS attacks. Indeed, the authentication sequence of PT is well designed to defend against DoS attacks. In the PT design, $R$ asks $I$ to verify $P I_{I}$ first, and then, $I$ asks $R$ to verify $P I_{R}$. If we transpose this sequence, attackers may send a lot of authentication requests to the targeted $R$ with forged $P I \mathrm{~s}$, each of whom asks $R$ to provide $(x, u) . R$ has to answer each request by computing $(x, u)$ and sending it back. $R$ then waits for challenges from these peers, which will never come. This kind of DoS attack may devour the computational resources or available network connections of $R$ and $T_{R}$ and render them unable to answer legitimate requests until the attack ends. To avoid this possible drawback, $P T$ orders that $I$ must prove itself to $R$ first, and then, $R$ conducts the verification of $I$. Therefore, if attackers conduct DoS attacks on $R$ by propagating numerous forged authentication requests, they must generate the same amount of valid PIs in advance. Otherwise, forged PIs would fail to pass the verification of $R$. For each verification request, $R$ does nothing but generate a random number $e$ for each $I$ before it completes the proving step. While $I$ has to own a valid $P I$ first and computes $(x, u)$ or, if possible, provides the answer $y$ to $R$ before asking $R$ to prove its $P I_{R}$. That is, the attackers should not only try to generate enough valid $P I$ s but also finish the proving phase on their side. Since the computational overhead in the verification process is relatively small compared to that in the proving procedure, a DoS attack hardly has an effective influence on $R$. Moreover, valid $P I$ s used to perform DoS attacks would degrade the reputations of those pseudo identities. To continue DoS attacks, adversaries have to generate more valid PIs. Even if adversaries form a clique to boost each other to corrupt the reputation system, the overhead of DoS attacks is quite expensive and unacceptable. Thus, the design of PT effectively defends against DoS attacks. 


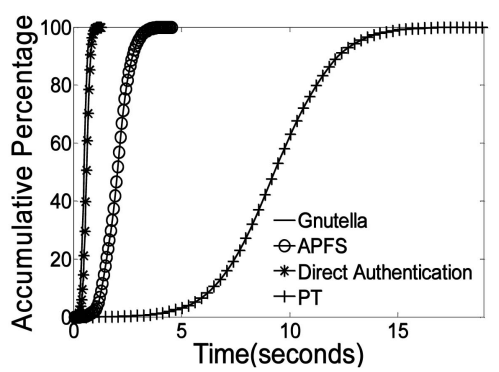

Fig. 5. Response time.

\section{Performance Evaluation}

We first evaluate the PT design by trace-driven simulations, in which the P2P topologies are obtained from the DSS Clip2 trace. Our simulations are performed on those traces in a variety of network sizes ranging from hundreds to thousands. For each simulation, we take the average result from 1,000 runs. The results are consistent with traces of different days, and here, we show the representative results.

P2P networks are highly dynamic, with peers frequently joining and leaving. We simulate the joining and leaving behavior of peers by turning on and off logical peers, respectively. In our simulation, each node issues 0.3 queries / $\mathrm{min}$. When a peer joins, a lifetime in seconds is assigned to the peer. The lifetime of a peer is defined as the time period that a peer stays in the system. The lifetime is generated according to the distribution observed in [24]. The mean of the distribution is chosen to be 10 minutes.

\subsection{Response Time}

Of all latencies in a P2P system, the response time from query issuance to the start of the download is of greatest concern, as it has a significant bearing on the system usability. Fig. 5 plots the simulation results of the response time, where we show the accumulative percentage of returned responses versus time for PT, overt Gnutella protocol, and APFS. Note that we have only included the latency of sending queries and responses in the APFS protocol, as we do not want the other components of APFS to influence our results.

The comparison in Fig. 5 shows that the response time of APFS is approximately three times that of overt Gnutella, while PT is around seven times that of overt Gnutella. In APFS, users need one onion path plus a flooding procedure to send a query out, one TCP link to deliver the response between tail nodes, and two onion paths to send the response anonymously. In $\mathrm{PT}^{\prime}$ s two-phase authentication procedures between two parties, the numbers of used onion paths and TCP links are 12 and 6 to follow APFS, respectively. According to the design of $\mathrm{PT}$, the authentication messages pass through the TCP connections between two tail nodes six times in a mutual authentication procedure. We also simulate a pure mutual authentication procedure without overt Gnutella and APFS, shown as the star line in Fig. 5. Our observation shows that the average response time of normal query flooding, direct authentication, APFS, and PT are about 493, 600, 2,031, and 9,296 ms, respectively. Note that the time consumed in anonymous paths of PT constitutes a major part of the whole latency,

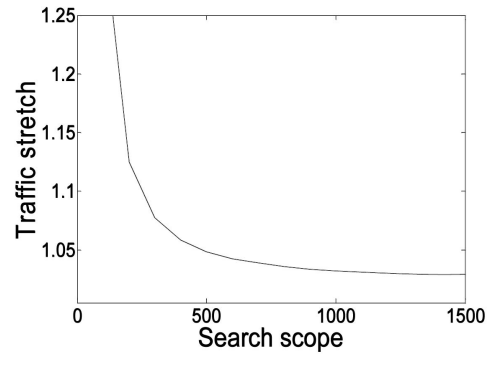

Fig. 6. Traffic stretch.

which is four times more than that of APFS. Therefore, the time consumption of authentication is indeed trivial. Our later offline implementations also support this summary.

\subsection{Traffic Overhead}

In our next experiment, we test the extra traffic cost brought about by authentication procedures. We define the traffic stretch as the traffic cost ratio between PT plus Gnutella and Gnutella only. As shown in Fig. 6, traffic stretch decreases when search scope increases. The traffic stretch is lower than 1.03 when the search scope reaches 1,400 peers, which means less than 3 percent additional traffic is incurred by PT.

The extra traffic cost is mainly incurred by anonymous communications and authentication interactions among peers. These connections comprise two anonymous sessions and a TCP link. Therefore, the scale of extra traffic cost mostly depends on the sum of those connection lengths. In fact, we also observe that the average distance between two random nodes tends to be constant with the growing size of the P2P overlay. As a result, the extra traffic cost caused by the PT authentication also slightly fluctuates around a constant. In our experiments, the traffic stretch first decreases sharply and then tends to be constant. As a reflection, Fig. 6 illustrates this tendency.

\subsection{Scalability}

As the size of the P2P overlay grows, PT has good scalability in the response time and the traffic overhead. This is due to the fact that 1) no central server or CA is involved during our authentication procedures, 2) the design is efficient and the extra computation needed is relatively trivial, and 3) the underlying anonymous protocol we have selected, APFS, has good scalability.

We increase the size of the P2P overlay from 500 to 13,000 and observe the average response time of 10,000 queries. Results show that the growth of the P2P overlay has little impact on the PT performance, as shown in Fig. 7. In fact, PT employs an authentication procedure through the direct TCP connection and the delay is mainly related to the average distance between two nodes in the physical Internet layer.

\section{Prototype Implementation}

We implement a prototype in our laboratories at the Chinese Academy of Sciences (CAS), Beijing, the campus of Hong Kong University of Science and Technology (HKUST), and other sites. We launched two-part experiments. 


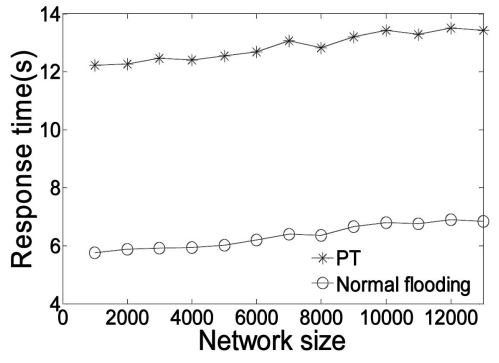

Fig. 7. Scalability of PT.

The first set focuses on the extra computation overhead caused by PT and tests the computing capabilities of normal PCs running this protocol. The second set tests the overall latency of pseudo identity authentication procedures in the Internet environment.

\subsection{Overview}

We develop PT prototype programs over VC6 and WinXP SP2 platforms. To shorten the development cycle, we use Victor Shoup's NTL [25], a number theory library, which has gained wide acceptance in the cryptography community for large integer operation.

The prototype P2P servant modifies Gnutella 0.6 by adding three major components: System initialization, Prover, and Verifier toolkit. The System initialization deals with new peers joining, including the $P I$ and PIC generation and issuance, parameters setup, anonymous path construction, and so on. The main authentication algorithms and their operations are conducted by the Prover and Verifier toolkit. To generate high-quality random and nondeterministic numbers, PT allows users to grab the system clock and mouse movements as the random seed resource.

\subsection{Offline Experiments}

We first examine the running capacity necessary for PT in an offline environment by conducting experiments on four different desktop PCs with the following configurations: PIII $450 \mathrm{M} / 128 \mathrm{M}$, PIV1.8G/256M, PIV2.6G/256M, and P-M1.4G/256M. Fig. 8 plots the time consumption of $P I C$ generation (including the $P I$ generation) on the above four machines with a 1,024-bit moduli, which is a default selection, providing enough security to PT. The PIC generation time grows linearly when the amount of quadratic residue, $k$, grows. In previous discussions, we show that it is safe when $k$ is no less than 80. For a current popular PC configuration, such generation needs

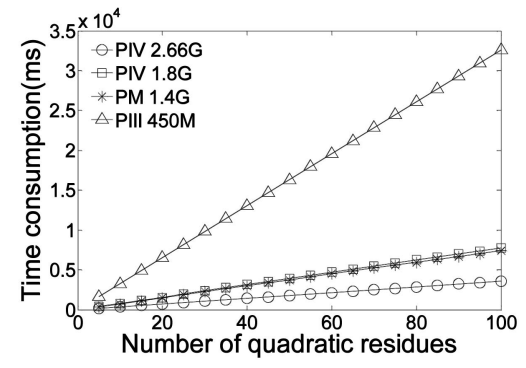

Fig. 8. Time consumption of $P I C$ generation.

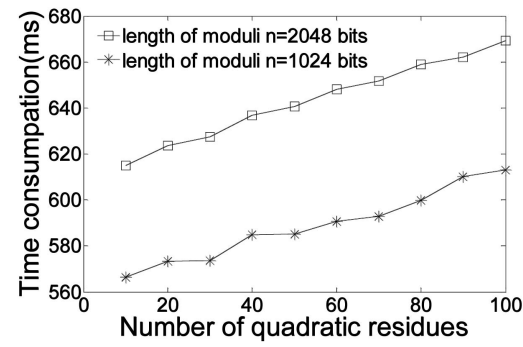

Fig. 9. Time consumption of authentication in the Internet.

less than 8 seconds, which is acceptable as PIC generation is a one-time job, necessary only when a peer joins the P2P community.

We also test the time consumption of the proving and verifying operations. The experiment demonstrates that the computation overhead of the authentication is trivial.

\subsection{Implementation in Internet Environments}

We then implement our PT prototype in an overlay comprising of 50 desktop PCs at the laboratories of CAS, the campus network of HKUST, and other sites. The representative configuration of each machine includes a PIV1.8G CPU, 256 MBytes of memory, and a 1000M Ethernet card.

To better evaluate PT, in this implementation, we ignore the time consumed by the APFS protocol. To easily adopt PT in current P2P systems, we have included some reliable modulus in API. The primary version of the PT package is available on our public Website [26].

Fig. 9 shows the experimental results in the Internet. The number of quadratic residues ranges from 10 to 100 , and we use 1,024 and 2,048 bits as moduli sizes, respectively.

We employ ping tests with packets of the same size as PT messages over the two involved computers between Hong Kong and Beijing in the Internet. The results range from 0.07 to 0.12 second. Therefore, the overall latency of PT is less than 0.67 second, which is relatively small for an authentication procedure. Note that the extra latency of PT in implementation results is much shorter than that in the simulation results because the time consumed by the Gnutella and APFS protocols is included in the simulation but not in the prototype implementation.

We also perform experiments in the campus area network and metropolitan area network. The experimental results can be found in our technical report [26]. To repeat our experiment, readers can find the package of PT prototype in [26].

\section{Conclusions}

Due to the inherent trade-off between trust and anonymity, identity-based trust management schemes cannot be directly employed in anonymous P2P systems. We propose an anonymous zero-knowledge authentication protocol, called PT. In this work, a ZKP-based authentication scheme is designed to support trust management in anonymous environments, so that peers may use unforgeable and verifiable pseudonyms instead of their real identities in P2P communities. 
We prove that the probability of a successful impersonation is computationally infeasible, even if the adversaries have collected all of the previous authentication messages. We also manage to defend against MIMAs. The results of trace-driven simulations show that $\mathrm{PT}$ is scalable in both static and dynamic environments. We also implement a prototype of PT and evaluate its performance through comprehensive experiments. We believe that wide deployment of this design will provide better privacy and security for P2P users.

\section{ACKNOWLEDGMENTS}

Some preliminary results of this paper were presented in the 2007 IEEE International Parallel and Distributed Processing Symposium. This research was supported in part by the National Science Foundation of China Grants 60573053 and 60673179, National Science Foundation of China Key Project 60736016, National Science Foundation of China Distinguished Young Scholar Grant 60525209, China 973 Fundamental R\&D Program 2005CB321803, China 863 National High Technology R\&D Program 2007AA01Z18, Hong Kong RGC Grant N_HKUST614/07 and HKUST 6152/06E, Microsoft Research Asia, and Nokia APAC Research Grant.

\section{REFERENCES}

[1] D. Qiu and R. Srikant, "Modeling and Performance Analysis of BitTorrent-Like Peer-to-Peer Networks," Proc. ACM SIGCOMM, 2004.

[2] W.W. Terpstra, J. Kangasharju, C. Leng, and A.P. Buchmann, "BubbleStorm: Resilient, Probabilistic, and Exhaustive Peer-toPeer Search," Proc. ACM SIGCOMM, 2007.

[3] Y. Liu, L. Xiao, and L.M. Ni, "Building a Scalable Bipartite P2P Overlay Network," IEEE Trans. Parallel and Distributed Systems, vol. 18, pp. 1296-1306, 2007.

[4] R. Sherwood, B. Bhattacharjee, and A. Srinivasan, "P5: A Protocol for Scalable Anonymous Communication," Proc. IEEE Symp. Security and Privacy (SEP), 2002.

[5] V. Scarlata, B.N. Levine, and C. Shields, "Responder Anonymity and Anonymous Peer-to-Peer File Sharing," Proc. Int'l Conf. Network Protocols (ICNP), 2001.

[6] P.P.C. Lee, J.C.S. Lui, and D.K.Y. Yau, “Distributed Collaborative Key Agreement and Authentication Protocols for Dynamic Peer Groups," IEEE/ACM Trans. Networking, vol. 14, pp. 263-276, 2006.

[7] E. Damiani, D.C.D. Vimercati, S. Paraboschi, P. Samarati, and F. Violante, "A Reputation-Based Approach for Choosing Reliable Resources in Peer-to-Peer Networks," Proc. ACM Conf. Computer and Comm. Security (CCS), 2002.

[8] S.D. Kamvar, M.T. Schlosser, and H. Garcia-Molina, "The EigenTrust Algorithm for Reputation Management in P2P Networks," Proc. Int'l Conf. World Wide Web (WWW), 2003.

[9] A. Abdul-Rahman and S. Halles, "A Distributed Trust Model," Proc. New Security Paradigms Workshop (NSPW), 1997.

[10] S. Lee, R. Sherwood, and B. Bhattacharjee, "Cooperative Peer Groups in NICE," Proc. IEEE INFOCOM, 2003.

[11] B. Dragovic, E. Kotsovinos, S. Hand, and P. Pietzuch, "XenoTrust: Event-Based Distributed Trust Management," Proc. IEEE Trust and Privacy in Digital Business Workshop (TrustBus), 2003.

[12] S. Goldwasser, S. Micali, and C. Rackoff, "The Knowledge Complexity of Interactive Proof Systems," SIAM J. Computing, vol. 18, pp. 186-208, 1989.

[13] J. Brandt, I.B. Damgard, P. Landrock, and T. Pedersen, "ZeroKnowledge Authentication Scheme with Secret Key Exchange," Proc. Advances in Cryptology (CRYPTO), 1990.

[14] O. Goldreich, Foundations of Cryptography. Cambridge Univ. Press, 2001.

[15] W. Mao, Modern Cryptography: Theory and Practice. Prentice Hall, 2004.
[16] U. Fiege, A. Fiat, and A. Shamir, "Zero Knowledge Proofs of Identity," Proc. ACM Conf. Theory of Computing (STOC), 1987.

[17] Digital Signature Standard, FIPS PUB 186, http://csrc.nist.gov/ publications/fips/fips186-2/fips186-2-change1.pdf, 2007.

[18] G. Ciaccio, "Improving Sender Anonymity in a Structured Overlay with Imprecise Routing," Proc. Privacy Enhancing Technologies Workshop (PET), 2006.

[19] N. Borisov, "Anonymous Routing in Structured Peer-to-Peer Overlays," PhD dissertation, Univ. of California, Berkeley, CA, 2005.

[20] A. Nambiar and M. Wright, "Salsa: A Structured Approach to Large Scale Anonymity," Proc. ACM Conf. Computer and Comm. Security (CCS), 2006.

[21] B. Schneier, Applied Cryptography_Protocols, Algorithms, and Source Coed in C, second ed. John Wiley \& Sons, Inc., 1996.

[22] J. Menezes, P.C.V. Oorschot, and S.A. Vanstone, Handbook of Applied Cryptography. CRC Press, 1996.

[23] K. Lenstra and E.R. Verheul, "Selecting Cryptographic Key Sizes," J. Cryptology, vol. 14, pp. 255-293, 2001.

[24] S. Saroiu, P. Gummadi, and S. Gribble, "A Measurement Study of Peer-to-Peer File Sharing Systems," Proc. Multimedia Computing and Networking (MMCN), 2002.

[25] NTL, http://shoup.net/ntl/, 2007.

[26] L. Lu, L. Hu, J. Li, J. Han, and Y. Liu, "Anonymity and Security Analysis of Pseudo Trust," technique report, http://www.cse.ust. hk/ liu/PseudoTrust.htm, 2007.

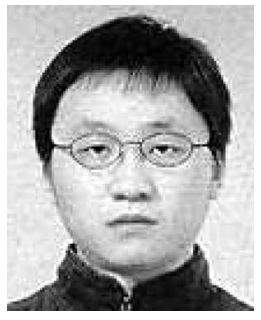

Li Lu received the BS and MS degrees from Zhejiang University, Hangzhou, China, in 2000 and 2003, respectively, and the PhD degree in information security from the Chinese Academy of Sciences, Beijing, in 2007. He is currently a postdoctoral fellow in the Department of Computer Science and Engineering, Hong Kong University of Science and Technology. His research interests include applied cryptography, network security, pervasive computing, and sensor networks. He is a member of the IEEE.

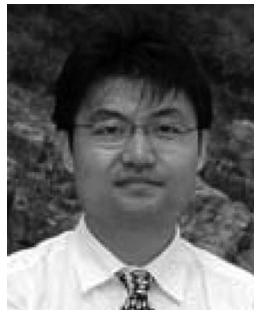

Jinsong Han received the BS degree in computer science from Shandong University of Technology, Zibo, China, in 1997, the MEng degree in computer science from Shandong University, Jinan, China, in 2000, and the PhD degree in computer science and engineering from Hong Kong University of Science and Technology (HKUST), in 2007. He is currently a postdoctoral fellow in the Department of Computer Science and Engineering, HKUST. His research interests include peer-to-peer computing, anonymity, network security, pervasive computing, and high-speed networking. $\mathrm{He}$ is a member of the IEEE, the IEEE Computer Society, and the ACM.

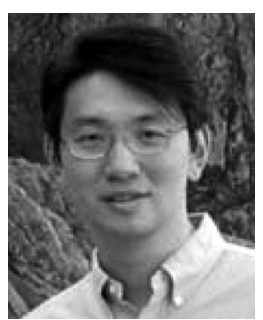

Yunhao Liu received the BS degree in automation from Tsinghua University, Beijing, in 1995, the MA degree from Beijing Foreign Studies University, in 1997, and the MS and $\mathrm{PhD}$ degrees in computer science and engineering from Michigan State University, East Lansing, in 2003 and 2004, respectively. He is currently with the Department of Computer Science and Engineering, Hong Kong University of Science and Technology. He is also an adjunct professor at Xi'an Jiaotong University, Xi'an, China. His research interests include peer-to-peer computing, pervasive computing, and sensor networks. He is a senior member of the IEEE, the IEEE Computer Society, and the ACM. 


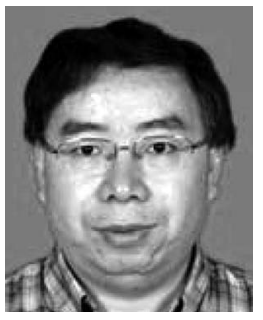

Lei Hu received the BS and MS degrees from Peking University, Beijing, in 1988 and 1991, respectively, and the $\mathrm{PhD}$ degree from the Chinese Academy of Sciences, Beijing, in 1994. Since 2002, he has been a professor at the Graduate University of the Chinese Academy of Sciences, Beijing. His research interest includes cryptology and information security.

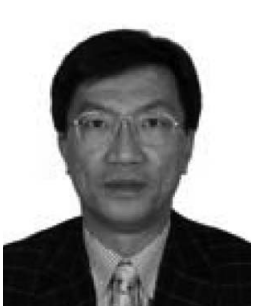

Jinpeng Huai is a professor and vice president of Beihang University. He serves on the Steering Committee for Information Technology, the National High-Tech Program (863) as the Chief Scientist. His research interests include serviceoriented computing and middleware, distributed computing, trustworthiness, and security. $\mathrm{He}$ has published more than 100 papers. He is a member of the IEEE.

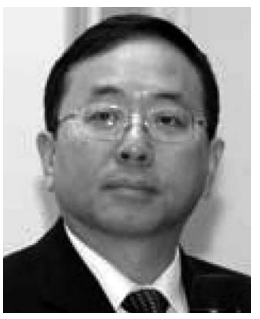

Lionel $\mathbf{M}$. Ni received the $\mathrm{PhD}$ degree in electrical and computer engineering from Purdue University, West Lafayette, Indiana, in 1980. He is a chair professor in the Department of Computer Science and Engineering, Hong Kong University of Science and Technology (HKUST). He is also the director of the HKUST China Ministry of Education/Microsoft Research Asia IT Key Laboratory and the director of the HKUST Digital Life Research Center. He has chaired many professional conferences and has received a number of awards for authoring outstanding papers. He is a fellow of the IEEE and the IEEE Computer Society.

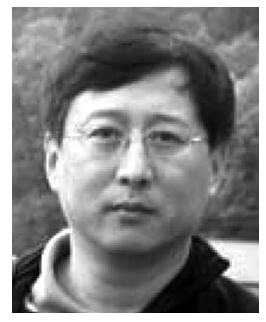

Jian Ma received the BSc and MSc degrees from the Beijing University of Posts and Telecommunications, in 1982 and 1987, respectively, and the PhD degree from Helsinki University of Technology, Espoo, Finland, in 1994. He is currently a principal member of research staff at Nokia Research Center, Beijing, where he is responsible for university cooperation in China and other APACs, Nokia Postdoc Working Station, and Nokia-Tsinghua Joint Research Laboratory. He has 11 patents granted and tens of patent applications. He is also an adjunct professor at Beijing University of Posts and Telecommunications; Institute of Computing Technology, Chinese Academy of Sciences; Graduate University of Chinese Academy of Sciences, Beijing; Tongji University, Shanghai; and Beihang University, Beijing. His current research interests are consumer services on wireless sensor networks, mobile Internet applications, pervasive computing, ad hoc and P2P networking, and so forth. He is the author or coauthor of more than 150 publications in journals and conference proceedings and a couple of books and book chapters.

$\triangleright$ For more information on this or any other computing topic, please visit our Digital Library at www.computer.org/publications/dlib. 